\title{
A Context for Training - Based Approximation in Randomly Correlated Rician Mimo Channels with Rician Disruption
}

\author{
${ }^{1}$ Kakileti Pradeep ${ }^{2}$ T. Aditya Kumar M.Tech \\ 126p1d3806Assistant Professor \\ Prasiddha College Of Engineering \& Technology Prasiddha College Of Engineering \&Technology
}

\begin{abstract}
An antenna array on the transmit side provides the system with an extra spatial dimension that can be utilized for coding both in the spatial as well as the temporal domain. The recent development of such space time codes shows that there are ways of exploiting multiple transmit antennas while completely avoiding traditional beam-forming techniques need of accurate channel state information. In this project, we generate a framework for training based channel estimation under different channel and interference statistics. The minimum mean square error (MMSE) estimator for channel matrix estimation in Rician fading multi-antenna systems is analyzed, and exclusively the proposal of mean square error (MSE) minimizing training structures. By considering Kronecker-structured systems with a grouping of noise and interference and random training sequence length, we gather and simplify numerous earlier results in the framework. We simplify the circumstances for attaining the optimal training sequence structure and show when the spatial training power distribution can be explained unambiguously. We also prove that spatial correlation improves the estimation performance and establish how it determines the optimal training sequence length. The analytic results for Kronecker-structured systems are used to derive a heuristic training sequence under general unstructured statistics. The MMSE estimator of the squared Frobenius norm of the channel matrix is also derived and shown to provide far better gain estimates than other approaches. It is shown under which conditions training sequences that minimize the non-convex MSE can be derived explicitly or with low complexity. Numerical examples are used to evaluate the performance of the two estimators for different training classifications and system statistics. We also elucidate how the finest length of the training sequence often can be shorter than the number of transmit antennas.
\end{abstract}

Keyword: mse, mmse estimator, kronecker-structured systems.

\section{Introduction}

In this paper, we consider training-based estimation ofinstantaneous CSI in multiple-input multipleoutput (MIMO)systems. Thus, the estimation is conditioned on the receivedsignal from a known training sequence, which potentially canbe adapted to the long-term statistics. By nature, the channel is stochastic, which motivates Bayesian estimation-that is, modeling of the current channel state as a realizationfrom a knownmulti-variate probability density function (PDF). There is also alarge amount of literature on estimation of deterministic MIMOchannels which are analytically tractable but in general provideless accurate channel estimates, as shown in [7], [8]. Herein,we concentrate on minimum mean square error (MMSE)estimation of the channel matrix and its squared Frobenius norm,given the first and second order system statistics.

Training-based MMSE estimation of MIMO channel matrices has previously been considered for KroneckerstructuredRayleigh fading systems that are either noise-limited [9]-[11]or interference-limited [12]. In these papers, optimization ofthe training sequence was considered under various limitations on the long-term statistics, and analogous structures of theoptimal training sequence were derived. These results reducethe training optimization to a convex power allocation problemthat can be solved explicitly in some special cases. Whenmentioning previous work, it is worth noting that simplifiedchannel matrix estimators have been developed in [8] and [13] and claimed to be MMSE estimators, but we show herein thatthese estimators are in general restrictive.

Although estimation of the channel matrix is important forreceive and transmit processing, knowledge of the squaredFrobenius norm of the channel matrix provides instantaneousgain information and can be exploited for rate adaptation andscheduling [17], [18]. The squared norm can be determinedindirectly from an estimated channel matrix, but as shown in[16] this approach gives poor estimation performance at mostsignalto-interference-and-noise ratios (SINRs). The MMSEestimator of the squared channel norm was introduced in [16]for Kronecker-structured Rayleigh fading channels, assumingthe same training structure as for channel matrix estimation.Herein, the estimator is proved and generalized to Rician fadingchannels, along with the 
design of MSE minimizing trainingsequences. Although the MSE is non-convex, we show thatthe optimal training sequence can be determined with limitedcomplexity.

\section{System Model}

We consider flat and block-fading MIMO systems with atransmitter equipped with an array of transmit antennasand a receiver with an array of receive antennas. Thesymbol-sampled complex baseband equivalent of theflatfading channel when transmitting at channel use is modelledas

$$
y(t)=H x(t)+n(t)
$$

A measure of the spatial channel correlation is the eigenvaluedistribution of the channel covariance matrix; weak correlationis represented by almost identical eigenvalues, while strongcorrelation means that a few eigenvalues dominate. Thus, in a highly correlated system, the channel is approximatelyconfined to a small eigensubspace, while all eigenvectors areequally important in an uncorrelated system. In urban cellularsystems, base stations are typically elevated and exposed tolittle near-field scattering. Thus, their antennas are strongly spatially correlated, while the non-line-of-sight mobile users areexposed to rich scattering and have weak antenna correlation ifthe antenna spacing is sufficiently large [19].

There are many reasons for estimating the channel matrixat the receiver. Instantaneous CSI can, for example, be used forreceive processing (improved interference suppression and simplified detection) and feedback (to employ beamforming andrate adaptation). In this section, we consider MMSE estimationof the channel matrix from the observation during training transmission. In general, the MMSE estimator of a vector from an observation is

$$
\hat{h}_{M M S E}=\mathrm{E}\{h / y\}=\int h f(h / y) d h
$$

We stress that the general MMSE estimator in (6) is in fact linear(affine), but nonetheless it has repeatedly been referred to as thelinear MMSE (LMMSE) estimator [10]-[12] which is correctbut could lead to the incorrect conclusion that there may existbetter non-linear estimators. The MMSE estimator in (6) is alsothe maximum a posteriori (MAP) estimator

Observe that the MSE depends on the training matrix and onthe covariance matrices of the channel and disturbance statistics, while it is unaffected by the mean values. Thus, the training matrix can potentially bedesigned to optimize the performance byadaptation to the second order statistics [9]-[12]. The intuitionbehind this training optimization is that more power should beallocated to estimate the channel in strong eigendirections (i.e.,large eigenvalues). Observe that training optimization is usefulin systems with dedicated training for each receiver, while multiuser systems with common training may require fixed or codebook-based training matrices(if users do not have the samechannel statistics).

For general channel and disturbance statistics, the MSE minimizing training matrix will not have any special formthat can beexploited when solving (9). However, if the covariance matricesand are structured, the optimal may inherit this structure.Previous work in training optimization has showed that in Kroneckerstructured systems with either noise-limited [9]-[11] orinterference-limited [12] disturbance, the optimal training matrix has a certain structure based on the transmit-side channelcovariance and temporal disturbance covariance. Herein, thisresult is generalized by showing that the same optimal structureappears in systems with both noise and interference. Then, wewill show how the training matrix behaves asymptotically andunder which conditions there exist explicit solutions to (9). Finally, we analyze how the statistics and total training power determines thesmallest length of the training sequence necessaryto achieve the minimal MSE. To summarize the results of this section, we have showedthe structure of the MSE minimizing training matrix in Kroneckerstructured systems and analyzed the allocation of powerbetween the eigendirections. Based on these results, we proposea heuristic training matrix that can be applied under generalsystem conditions. Observe that even whenKronecker-structured approximations are used in the training sequence design, the general MMSE estimator in (6) should always be appliedwithout these approximations.

\section{Mmse Estimation Of Squared Channel Norms}

In many applications, it is of great interest to estimate thesquared Frobenius norm of the channel matrix. This normcorresponds directly to the SINR in space-time block coded(STBC) systems and has a large impact on the SINR in manyother types of systems [7], [8]. The channel norm can be estimated indirectly from anestimated channel matrix, for exampleusing the estimator in (6). This will however lead to suboptimal performance and gives poor estimates at low training power. Thus, we consider training-based MMSE estimation of in this section

Analysis of the squared channel norm is considerably moreinvolved than for the channel matrix. The next theorem gives ageneral expression for the MMSE estimator and its MSE, andspecial expressions for Kronecker-structured systems. In orderto derive these expressions, we limit the analysis to training 
A context for training - based approximation in randomly correlated rician mimo channels...

matrices with the structure. It is our conjecture that the MSE minimizing training matrix has this form,3aswas proved in Theorem 1 for channel matrix estimation. Thistraining matrix structure is also of most practicalimportance,since the same training signalling will be used to estimate..low training power can be derived explicitly. Observe that theMSE depends on the mean value of the channel, while theMSE for channel matrix estimation is independent of the mean.The limiting solutions are however similar in the sense that allpower is allocated in a single Eigen direction at low power andare spread in all spatial direction at high power. The definition of the strongest direction at low training power and theproportional power distribution at largepower are however different, which means that the MSE minimizing training matricesusually are different for matrix and squared norm estimation.

In this section, the performance of the MMSE estimators andthe training sequence design will be illustrated numerically. TheMSE performance of the channel matrix estimator was thoroughly evaluated in [12] for interference-limited Kroneckerstructured systems. Thus, we consider the opposite setting ofa noise-limited non-Kronecker-structured system, and we willcompare the MMSE estimation performance with other recently proposed estimators. This section will also illustrate the advantage of direct MMSE estimation of the squaredchannel normover indirect calculation from an estimated channel matrix. Finally, we will illustrate how the smallest necessary length of thetraining sequence depends on the spatial correlation and available training power.

\section{OUTPUTS}

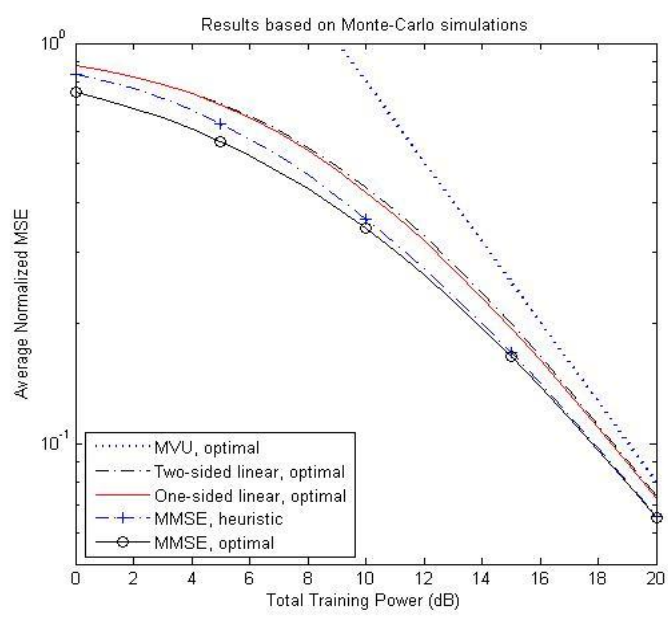

Fig 1The normalized MSEs of channel matrix estimation as a function of the total training power in a system with the Weichselberger model and the coupling matrix proposed

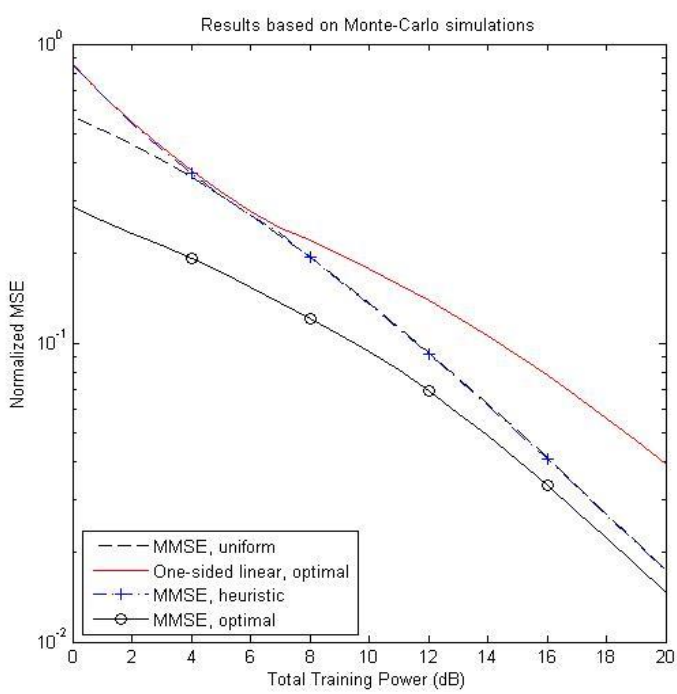

Fig 2The normalized MSEs of channel squared norm estimation as a function of the total training power in a system with uncorrelated receive antennasand a transmit antenna correlation of 2.4. 


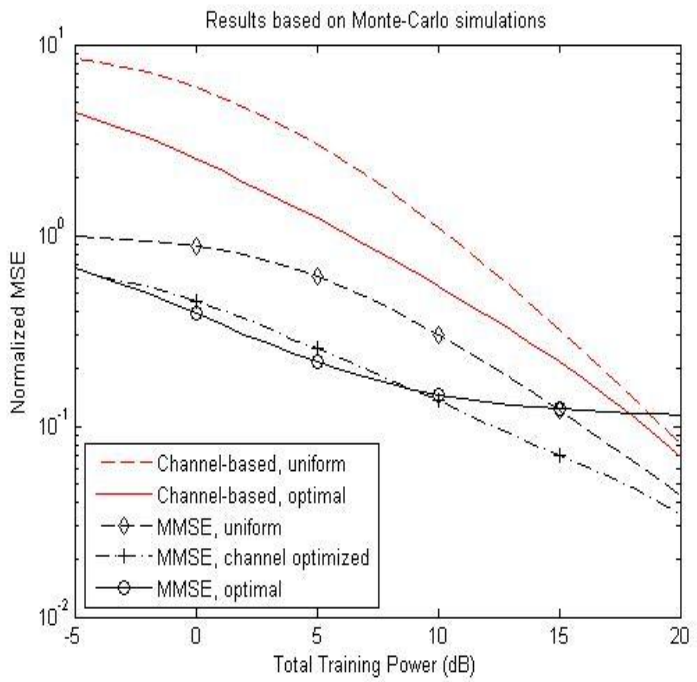

Fig 3The MMSE estimator with three differenttraining matrices is compared with the one-sided linear estimator

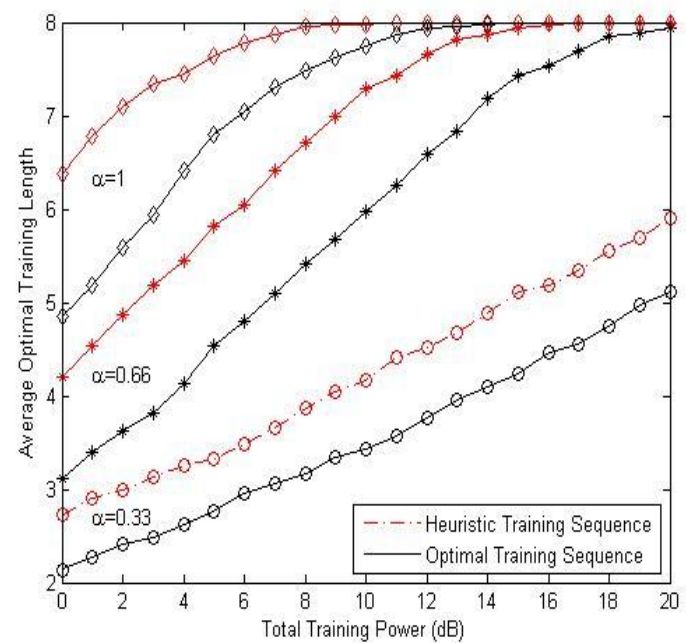

Fig 4 The average optimal training sequence length (smallest length that minimizes the MSE) as a function of the total training power

\section{Conclusion}

The performance analysis given throughout this thesis is based on a large collection of new randommatrix theory results, which were presented. The key utility of theseresults is that, in contrast to many existing results in random matrix theory, they involve simplefinite expressions, and can be easily and efficiently evaluated. This is quite remarkable for manyof the results, such as those involving noncentral matrix-variate quadratic forms, given the hugecomplexity of the underlying matrix-variate distributions. The capacity were derived based on the new random determinant propertiesof complex noncentralWishart matrices and matrixvariate quadratic formsThe properties for the Wishart case were derived using matrix-variate integrals and various determinant operations, as well as a new determinant representation for the classical hypergeometricfunction $1 \mathrm{~F}^{\sim} 1(\cdot)$ of a single complex matrix argument,. The properties for thequadratic form case were derived by first using determinant operations to re-formulate the problems into ones involving only Wishart matrices, and then solving these with the help of the Wishartproperties. The ergodic capacity bounds were initially obtained using two classical inequalitiesfrom information theory due to Jensen and Minkowski. In order to evaluate the bounds in closedform however, using the results of, it was first necessary to re-formulate the requiredexpectations using am.g.f. approach. A similar method was also employed to obtain the capacityvariance results.The capacity results were derived based on the new expression for the joint unordereddistribution of two (jointly) correlated complex Wishart matrices, given. This wasobtained by marginalizing a joint eigenvalue distribution, using some determinant expansions. The performance results in Chapter 5 were derived based on the new expressions for the maximumeigenvalue distribution of complex central matrix-variate quadratic forms, given.The derivations in this case were particularly involved. 
Specifically, these results were derivedby directly marginalizing the joint eigenvalue distribution given in, which involvea $0 \mathrm{~F}^{\sim} \mathrm{O}(\cdot)$ hypergeometric function of three matrix arguments; for which there is no equivalentdeterminant representation. Thus the marginalization was required to be carried out using thevery complicated zonal polynomial infinite series representation given. The key toolsused for handling this expression were advanced Vandermonde determinant operations, and theCauchy-Binet Theorem (this was employed for re-summing the infinite series at the end).

\section{Reference}

[1]. B. Hassibi and B. Hochwald, "How much training is needed in multiple-antenna wireless links?," IEEE Trans. Inf. Theory, vol. 49, no. 4,pp. 951-963, 2003.

[2]. J. Pang, J. Li, L. Zhao, and Z. Lü, "Optimal training sequences forMIMO channel estimation with spatial correlation," in Proc. IEEEVTC’07-Fall, 2007, pp. 651-655.

[3]. E. Björnson and B. Ottersten, "Training-based Bayesian MIMOchannel and channel norm estimation," in Proc. IEEE ICASSP'09,2009, pp. 2701-2704.

[4]. E. Björnson, D. Hammarwall, and B. Ottersten, "Exploiting quantizedchannel norm feedback through conditional statistics in arbitrarily correlated MIMO systems,” IEEE Trans. Signal Process., vol. 57, no. 10,pp. 4027-4041, 2009.

[5]. X. Zhang, E. Jorswieck, and B. Ottersten, "User selection schemesin multiple antenna broadcast channels with guaranteed performance,"presented at the IEEE SPAWC'07, Helsinki, Finland, Jun. 17-20, 2007.

[6]. R. Ertel, P. Cardieri, K. Sowerby, T. Rappaport, and J. Reed, "Overviewof spatial channel models for antenna array communication systems,’IEEE Personal Commun. Mag., vol. 5, pp. 10-22, 1998.

[7]. E. Jorswieck and H. Boche, "Majorization and matrix-monotone functions in wireless communications," Foundations and Trends in Communication and Information Theory, vol. 3, pp. 553-701, 2007.

[8]. E. Björnson, E. Jorswieck, and B. Ottersten, "Impact of spatial correlation and precoding design in OSTBC MIMO systems," IEEE Trans. Wireless Commun., submitted for publication.

A. Marshall and I. Olkin, Inequalities: Theory of Majorization and ItsApplications. Boston, MA: Academic Press, 1979

[9]. Tulino, A. Lozano, and S. Verdú, "Impact of antenna correlation the capacity of multiantenna channels," IEEE Trans. Inf. Theory, vol.51, no. 7, pp. 2491-2509, 2005. W. Weichselberger, M. Herdin, H. Özcelik, and E. Bonek, "A stochastic MIMO channel model with joint correlation of both link ends,'IEEE Trans. Wireless Commun., vol. 5, no. 1, pp. 90-100, 2006.

[10]. W. Hager, Y. Liu, and T. Wong, "Optimization of generalized meansquare error in signal processing and communication," Linear Algebraand Its Applications, vol. 416, pp. 815-834, 2006. 